\title{
The counterattack of anti-HER2 small molecule compounds has started
}

\author{
Toshinari Yamashita \\ Department of Breast and Endocrine Surgery, Kanagawa Cancer Center, Yokohama, Japan \\ Correspondence to: Toshinari Yamashita, MD, PhD. Department of Breast and Endocrine Surgery, Kanagawa Cancer Center, 2-3-2 Nakao, Asahi-ku, \\ Yokohama 241-8515, Japan. Email: tyamashita@kcch.jp. \\ Provenance and Peer Review: This article was commissioned by the editorial office, Translational Breast Cancer Research. The article did not undergo \\ external peer review. \\ Comment on: Yan M, Bian L, Hu X, et al. Pyrotinib plus capecitabine for human epidermal growth factor receptor 2-positive metastatic breast cancer \\ after trastuzumab and taxanes (PHENIX): a randomized, double-blind, placebo-controlled phase 3 study. Transl Breast Cancer Res 2020;1:13.
}

Received: 05 July 2020; Accepted: 15 July 2020; Published: 30 July 2020.

doi: $10.21037 /$ tbcr-20-35

View this article at: http://dx.doi.org/10.21037/tbcr-20-35

There are two primary types of drugs that target human epidermal growth factor receptor 2 (HER2). One is an antibody-based drug against the HER2 protein and the other is a small molecule compound that inhibits phosphorylation of the HER2 intracellular domain. For many years, antibody-based therapies have been central to the treatment of HER2-positive breast cancer. It is well known that trastuzumab has dramatically changed the treatment for HER2-positive breast cancer and its prognosis (1). Lapatinib, which became available after trastuzumab, also inhibited HER1 phosphorylation and was initially expected to be effective even against HER2negative disease. Therefore, a study including HER2negative advanced recurrent breast cancer was conducted, but the results demonstrated effectiveness only for HER2positive breast cancer (2). The MA.31 trial comparing trastuzumab or lapatinib with taxanes as first-line treatment for HER2-positive advanced recurrence found that lapatinib was inferior to trastuzumab (3). Lapatinib never replaced trastuzumab for first-line treatment. Lapatinib in combination with trastuzumab improved progression free survival (PFS) compared to lapatinib alone, but the improvement was modest so it was positioned as a palliative treatment with few side effects (4). In the NeoALTTO trial using paclitaxel plus either lapatinib or trastuzumab or both for patients with HER2-positive preoperative breast cancer, the pCR (pathological complete response) rate was significantly increased in patients receiving combined trastuzumab and lapatinib (5). However, the parallel ALTTO study did not show a significant improvement in DFS (disease free survival) in postoperative cases and was not used for perioperative treatment (6).

The next agent used in combination therapy with trastuzumab was not a small molecule compound, but pertuzumab, which is a similar anti-HER2 antibody that emerged after lapatinib and took its place. The CLEOPATRA study used the combination as a firstline treatment for advanced recurrence of HER2-positive breast cancer and both PFS and overall survival were significantly prolonged (7). Trastuzumab, pertuzumab and taxane combination therapy is the standard regimen for first-line HER2-positive breast cancer. In addition, in the APHINITY study, pertuzumab added to trastuzumab plus a taxane showed improvement compared with conventional trastuzumab and taxane combinations, especially in nodepositive cases (8). This combination is used in perioperative treatment of breast cancer with high HER2-positive recurrence risk.

T-DM1, a trastuzumab-based antibody-drug conjugate that was developed around the same time as pertuzumab, showed significantly better PFS and OS than lapatinib plus capecitabine in the EMILIA study (9). This study positioned the small molecule compound lapatinib in a later therapeutic line. In the MARIANNE study, which is a firstline treatment for advanced and recurrent disease, T-DM1 had similar results to trastuzumab plus docetaxel (10). 
Since T-DM1 is equivalent to trastuzumab and docetaxel combination therapy, it may be less effective than trastuzumab, pertuzumab and taxane combination therapy. However, because of its tolerable side effects, it has established itself as a second-line treatment for advanced recurrent breast cancer. Furthermore, in the KATHERINE study, T-DM1 was used in place of trastuzumab and pertuzumab for patients who did not achieve pCR by preoperative therapy, and it was shown that recurrence was reduced by half, and the indication is expanding to the perioperative setting (11). In the ATEMPT trial, which compared trastuzumab and paclitaxel combination therapy with T-DM1 as postoperative treatment for stage 1 HER2positive breast cancer, both treatments were almost the same (12). In addition, trastuzumab deruxtecan (DS8201) is an antibody-drug conjugate composed of an antiHER2 antibody, a cleavable tetrapeptide-based linker, and a cytotoxic topoisomerase I inhibitor. It showed a very good result with a response rate of $60.9 \%$ and a median PFS of 16.4 months in patients who had previously been heavily treated (13). Trastuzumab deruxtecan is used in the United States and Japan and it is positioned as the thirdline treatment after T-DM1 use, but is currently being compared with T-DM1 in the DESTINY-Breast03 trial. In addition, trastuzumab deruxtecan is expected to undergo clinical trials aiming for use in earlier treatment lines, in which case drug-induced interstitial pneumonia may be an obstacle.

Neratinib is a small molecule compound other than lapatinib, and is known to widely and irreversibly inhibit pan-ErbB receptor. Although it did not show a significant result as a treatment for advanced recurrence, it has been shown to reduce recurrence especially for ER-positive breast cancer by adding it in cases where postoperative trastuzumab treatment is completed (14).

Pyrotinib, the subject of the current report, is also a promising small molecule compound. It is an irreversible pan-ErbB receptor tyrosine kinase inhibitor targeting HER1, HER2, and HER4. PHENIX was a randomized, double-blinded, placebo-controlled, multicenter phase 3 trial reported by Yan et al. This is a trial comparing capecitabine and placebo with capecitabine and pyrotinib combination therapy in patients with HER2-positive advanced relapse treated with trastuzumab and a taxane. The median PFS with capecitabine and placebo was 4.1 months, whereas the combination of capecitabine and pyrotinib significantly increased PFS to 11.1 months. The hazard ratio shows a very high effect of 0.18 . In a study evaluating the combination of capecitabine and lapatinib, the median PFS of capecitabine alone was 4.4 months and the median PFS of capecitabine and lapatinib combination therapy was 8.4 months [0.49 (95\% confidence interval, 0.34 to 0.71 ) (15). The improvement effect seemed to be better when combined with pyrotinib. In a study of capecitabine and lapatinib, about half of patients had advanced recurrence after second-line treatment and a large proportion of lines were slightly later than for PHENIX, but the median PFS of capecitabine was almost the same in both trials. The PHENIX trial is not a direct comparison with lapatinib, but the results of this study also suggest that pyrotinib is a promising drug. Moreover, the pyrotinib group had good results with a statistically significant difference $(\mathrm{P}<0.001)$ at 18.1 months [HR, $0.36(95 \% \mathrm{CI}$, 0.23 to 0.58$)$ ]. In the PHENIX study, a sequential study using single-agent pyrotinib was set up for patients who progressed in the control group of capecitabine and placebo, and median PFS of 5.5 months and an ORR of $38.0 \%$ were achieved. These results tend to be better than the previously reported data for lapatinib alone and neratinib alone. The use of pyrotinib alone or in combination with other anticancer agents is also promising.

Results of trials comparing pyrotinib directly with lapatinib have also been reported. A randomized, phase II study comparing pyrotinib or lapatinib combined with capecitabine for HER2-positive metastatic breast cancer patients previously treated with taxanes, anthracyclines, and/or trastuzumab was reported. Sixty-five were assigned to the pyrotinib group and 63 to the lapatinib group, with a median PFS of 18.1 months for the pyrotinib group and 7 months for the lapatinib group, which was significantly superior for the pyrotinib group (16). The results of the phase 3 PHOEBE trial of the same design were reported at the 2020 ASCO Annual Meeting. Two hundred and sixtyseven individuals were assigned to the pyrotinib or lapatinib arms in a 1:1 ratio and compared. The median PFS was 12.5 months (95\% CI, 9.7-not reached) with pyrotinib plus capecitabine versus 6.8 months (95\% CI, 5.4-8.1) with lapatinib plus capecitabine (HR, 0.39; 95\% CI, 0.27-0.56; $\mathrm{P}<0.0001)$, which was a statistically significant result $(\leq 0.0066)(17)$.

Tucatinib, orally presented at the 2019 SABCS, is also a promising anti-HER2 small molecule compound and it is highly selective in inhibiting HER2, so that adverse events such as diarrhea are less severe than for lapatinib and neratinib. The combination of placebo and tucatinib with trastuzumab and capecitabine significantly improved the 
median PFS in the tucatinib group compared to 5.6 months in the placebo group at 7.8 months [HR; 0.54 (95\% CI, 0.42-0.71)] (18). Furthermore, a significant improvement in overall survival [HR; 0.66 (95\% CI, 0.50-0.88); $\mathrm{P}=0.005$ ] was observed, and the FDA recently approved its use.

Treatment for brain metastasis is important in HER2positive breast cancer. HER2-positive breast cancer is the most likely type to metastasize to the brain, and the prognosis for cases with brain metastases is poor (19). However, among brain metastases, HER2-positive breast cancer patients have been reported to have longer survival times compared to other intrinsic subtypes (20). Small molecule compounds have a small molecular weight and penetrate the blood brain barrier, so they are expected to be effective for brain metastasis. The LANDSCAPE study using lapatinib and capecitabine was performed in patients with brain metastases (21). Although an effect was observed, the effect was not sustained and brain metastatic lesions progressed. Moreover, it was not recommended from the viewpoint that Grade 3 side effects appear in $50 \%$ of patients and the QOL decreases. In the TBCRC 022 trial, neratinib also had a CNS ORR of $49 \%$ in the volumetric response and an ORR of $24 \%$ for the total major axis (22). The NALA trial was a phase 3 trial comparing neratinib and capecitabine to lapatinib and capecitabine, with the neratinib group showing better PFS results $(\mathrm{HR}=0.76$; 95\% CI, 0.63-0.93; $\mathrm{P}=0.006$ ) (23). Time to intervention for symptomatic CNS disease (overall cumulative incidence $22.8 \%$ vs. $29.2 \% ; \mathrm{P}=0.043$ ) was delayed with neratinib and capecitabine vs. lapatinib and capecitabine. The PHENIX trial using pyrotinib also included cases of brain metastases, but the median PFS was 6.9 months for the combined pyrotinib and capecitabine arm and 4.2 months for the capecitabine and placebo arm, showing better results with the combination of pyrotinib. Moreover, the appearance of new brain metastases was $1.2 \%$ in the pyrotinib and capecitabine arm and $3.6 \%$ in the capecitabine and placebo arm, and it is possible that pyrotinib suppressed the progress and development of brain metastases.

Until now, antibody-based therapy has been in the limelight for the treatment of HER2-positive breast cancer, but small molecule compounds will also share the limelight in the near future. Using anti-HER2 antibodies in postoperative treatment has reduced the rate of recurrence, but the overall incidence of brain metastases has not been reduced $(8,24)$. Therefore, the importance of small molecule compounds that reduce rates of brain metastasis as a treatment for HER2-positive advanced recurrent breast cancer will increase. It may be possible to suppress the development and progression of brain metastases by using a HER2 small molecule compound in the perioperative period and in the early stage of recurrence. The HER2 CLIMB-2 trial to consider tucatinib in combination with T-DM1 has been initiated. Based on the results of PHENIX trial, it is considered that pyrotinib will be used in even earlier lines in the future. Small molecule compounds have the potential to suppress recurrence of brain metastases themselves if used during the perioperative period.

In the treatment of HER2-positive breast cancer, which had previously been centered on antibody-based drugs, the counterattack of small molecule compounds has just begun.

\section{Acknowledgments}

Funding: None.

\section{Footnote}

Conflicts of Interest: The author has completed the ICMJE uniform disclosure form (available at http://dx.doi. org/10.21037/tbcr-20-35). TY received honoraria for lectures held by Chugai, Eisai, Novartis, Taiho, Nippon Kayaku, AstraZeneca, Kyowa-Kirin, Pfizer, Eli Lilly, Daiichi-Sankyo Institutional and research funding from Chugai, Taiho, Nippon Kayaku, Kyowa-Kirin.

Ethical Statement: The author is accountable for all aspects of the work in ensuring that questions related to the accuracy or integrity of any part of the work are appropriately investigated and resolved.

Open Access Statement: This is an Open Access article distributed in accordance with the Creative Commons Attribution-NonCommercial-NoDerivs 4.0 International License (CC BY-NC-ND 4.0), which permits the noncommercial replication and distribution of the article with the strict proviso that no changes or edits are made and the original work is properly cited (including links to both the formal publication through the relevant DOI and the license). See: https://creativecommons.org/licenses/by-nc-nd/4.0/.

\section{References}

1. Dawood S, Broglio K, Buzdar AU, et al. Prognosis of women with metastatic breast cancer by HER2 status and trastuzumab treatment: an institutional-based review. J 
Clin Oncol 2010;28:92-8.

2. Di Leo A, Gomez HL, Aziz Z, et al. Phase III, doubleblind, randomized study comparing lapatinib plus paclitaxel with placebo plus paclitaxel as first-line treatment for metastatic breast cancer. J Clin Oncol 2008;26:5544-52.

3. Gelmon KA, Boyle FM, Kaufman B, et al. Lapatinib or Trastuzumab Plus Taxane Therapy for Human Epidermal Growth Factor Receptor 2-Positive Advanced Breast Cancer: Final Results of NCIC CTG MA.31. J Clin Oncol 2015;33:1574-83.

4. Blackwell KL, Burstein HJ, Storniolo AM, et al. Overall survival benefit with lapatinib in combination with trastuzumab for patients with human epidermal growth factor receptor 2-positive metastatic breast cancer: final results from the EGF104900 Study. J Clin Oncol 2012;30:2585-92.

5. Baselga J, Bradbury I, Eidtmann H, et al. Lapatinib with trastuzumab for HER2-positive early breast cancer (NeoALTTO): a randomised, open-label, multicentre, phase 3 trial. Lancet 2012;379:633-40.

6. Piccart-Gebhart M, Holmes E, Baselga J, et al. Adjuvant Lapatinib and Trastuzumab for Early Human Epidermal Growth Factor Receptor 2-Positive Breast Cancer: Results From the Randomized Phase III Adjuvant Lapatinib and/ or Trastuzumab Treatment Optimization Trial. J Clin Oncol 2016;34:1034-42.

7. Swain SM, Baselga J, Kim SB, et al. Pertuzumab, trastuzumab, and docetaxel in HER2-positive metastatic breast cancer. N Engl J Med 2015;372:724-34.

8. von Minckwitz G, Procter M, de Azambuja E, et al. Adjuvant Pertuzumab and Trastuzumab in Early HER2Positive Breast Cancer. N Engl J Med 2017;377:122-31.

9. Diéras V, Miles D, Verma S, et al. Trastuzumab emtansine versus capecitabine plus lapatinib in patients with previously treated HER2-positive advanced breast cancer (EMILIA): a descriptive analysis of final overall survival results from a randomised, open-label, phase 3 trial. Lancet Oncol 2017;18:732-42.

10. Perez EA, Barrios C, Eiermann W, et al. Trastuzumab Emtansine With or Without Pertuzumab Versus Trastuzumab Plus Taxane for Human Epidermal Growth Factor Receptor 2-Positive, Advanced Breast Cancer: Primary Results From the Phase III MARIANNE Study. J Clin Oncol 2017;35:141-8.

11. von Minckwitz G, Huang CS, Mano MS, et al. Trastuzumab Emtansine for Residual Invasive HER2Positive Breast Cancer. N Engl J Med 2019;380:617-28 12. Tolaney SM, Trippa L, Barry W, et al. Abstract GS1-05:
TBCRC 033: A randomized phase II study of adjuvant trastuzumab emtansine (T-DM1) vs paclitaxel (T) in combination with trastuzumab $(\mathrm{H})$ for stage I HER2positive breast cancer (BC) (ATEMPT). Cancer Res 2020;80:Abstract nr GS1-05. doi: 10.1158/1538-7445. SABCS19-GS1-05.

13. Modi S, Saura C, Yamashita T, et al. Trastuzumab Deruxtecan in Previously Treated HER2-Positive Breast Cancer. N Engl J Med 2020;382:610-21.

14. Martin M, Holmes FA, Ejlertsen B, et al. Neratinib after trastuzumab-based adjuvant therapy in HER2-positive breast cancer (ExteNET): 5-year analysis of a randomised, double-blind, placebo-controlled, phase 3 trial. Lancet Oncol 2017;18:1688-700.

15. Geyer CE, Forster J, Lindquist D, et al. Lapatinib plus capecitabine for HER2-positive advanced breast cancer. N Engl J Med 2006;355:2733-43.

16. Ma F, Ouyang Q, Li W, et al. Pyrotinib or lapatinib combined with capecitabine in HER2-positive metastatic breast cancer with Prior taxanes, anthracyclines, and/or trastuzumab: a randomized, phase II study. J Clin Oncol 2019;37:2610-9.

17. Xu B, Yan M, Ma F, et al. Pyrotinib or lapatinib plus capecitabine for HER2 + metastatic breast cancer (PHOEBE): a randomized phase III trial 2020 ASCO Virtual Scientific Program. Available online: https:// meetinglibrary.asco.org/record/184810/abstract

18. Murthy RK, Loi S, Okines A, et al. Tucatinib, Trastuzumab, and Capecitabine for HER2Positive Metastatic Breast Cancer. N Engl J Med 2020;382:597-609.

19. Brufsky AM, Mayer M, Rugo HS et al. Central nervous system metastases in patients with HER2-positive metastatic breast cancer: incidence, treatment, and survival in patients from registHER. Clin Cancer Res 2011;17:4834-43.

20. Darlix A, Louvel G, Fraisse J, et al. Impact of breast cancer molecular subtypes on the incidence, kinetics and prognosis of central nervous system metastases in a large multicentre real-life cohort. Br J Cancer 2019;121:991-1000.

21. Bachelot T, Romieu G, Campone M, et al. Lapatinib plus capecitabine in patients with previously untreated brain metastases from HER2-positive metastatic breast cancer (LANDSCAPE): a single-group phase 2 study. Lancet Oncol 2013;14:64-71.

22. Freedman RA, Gelman RS, Anders CK, et al. TBCRC 022: A Phase II Trial of Neratinib and Capecitabine for Patients With Human Epidermal Growth Factor Receptor 
2-Positive Breast Cancer and Brain Metastases. J Clin Oncol 2019;37:1081-9.

23. Saura C, Oliveira M, Feng YH, et al. Neratinib + capecitabine versus lapatinib + capecitabine in patients with HER2 + metastatic breast cancer previously treated with $\geq 2$ HER2-directed regimens: Findings from the multinational, randomized, phase III NALA trial. J Clin

doi: 10.21037/tbcr-20-35

Cite this article as: Yamashita T. The counterattack of antiHER2 small molecule compounds has started. Transl Breast Cancer Res 2020;1:10.
Oncol 2019;37:1002.

24. Pestalozzi BC, Holmes E, de Azambuja E, et al. CNS relapses in patients with HER2-positive early breast cancer who have and have not received adjuvant trastuzumab: a retrospective substudy of the HERA trial (BIG 1-01). Lancet Oncol 2013;14:244-8. 